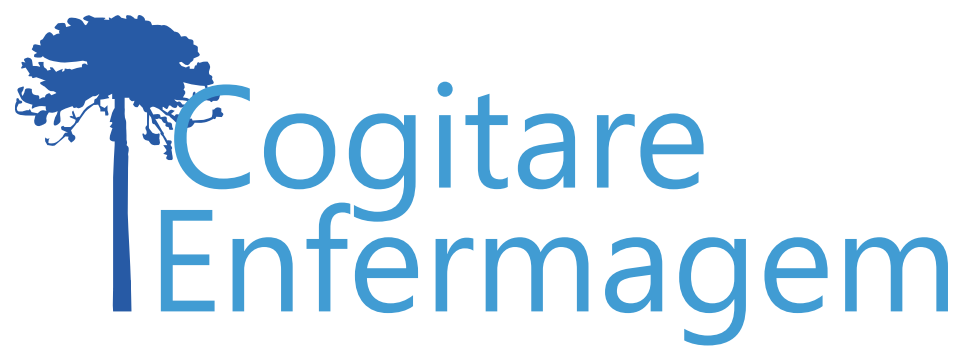

REVISÃO

\title{
DIMENSÕES QUE INTERFEREM NA ADESÃO À MEDICAÇÃO NOS TRANSTORNOS RELACIONADOS ÀS SUBSTÂNCIAS: REVISÃO INTEGRATIVA*
}

\author{
Fernanda Carolina Capistrano', Mariluci Alves Maftum² ${ }^{2}$ Camila Bonfim de Alcântara ${ }^{3}$, Aline \\ Cristina Zerwes Ferreira ${ }^{4}$, Gustavo Jorge Maftum ${ }^{5}$
}

\section{RESUMO}

Objetivo: Analisar a produção científica acerca das dimensões que interferem na adesão à terapêutica medicamentosa de pessoas com transtornos mentais relacionados ao uso de substância, no período de 2006 a abril de 2017.

Método: Revisão Integrativa da Literatura a partir de três bancos de dados, LILACS, CINAHL e PUBMED, realizada em abril de 2017.

Resultados: Foram analisados 43 estudos que atendiam aos critérios de elegibilidade. A análise dos estudos possibilitou identificar fatores que impactam positiva ou negativamente a adesão à terapêutica medicamentosa, agrupados de acordo com as cinco dimensões determinantes para a adesão proposta pela Organização Mundial da Saúde, sendo elas: socioeconômica, equipe/ sistema de saúde, doença, tratamento, e indivíduo/paciente.

Conclusão: Embora a terapêutica medicamentosa seja imprescindível no gerenciamento da pessoa com transtorno relacionado a substâncias, muitas vezes representa um desafio devido às diferentes dimensões interativas que influenciam diretamente no processo de adesão.

DESCRITORES: Adesão a medicação; Cooperação do paciente; Tratamento farmacológico; Transtornos Relacionados ao Uso de Substâncias; Saúde mental.

\footnotetext{
*Artigo extraído da tese de doutorado "Adesão à terapêutica medicamentosa por pessoas em tratamento em centros de atenção psicossocial - álcool e drogas". Universidade Federal do Paraná, 2018.
}

COMO REFERENCIAR ESTE ARTIGO:

Capistrano FC, Maftum MA, Alcântara CB de, Ferreira ACZ, Maftum GJ. Dimensões que interferem na adesão à medicação nos transtornos relacionados às substâncias: revisão integrativa. Cogitare enferm. [Internet]. 2019 [acesso em "colocar data de acesso, dia, mês abreviado e ano"]; 24. Disponível em: http://dx.doi.org/10.5380/ ce.v24i0.58170.

Este obra está licenciado com uma Licença Creative Commons Atribuição 4.0 Internacional.

${ }^{1}$ Enfermeira. Doutoranda em Enfermagem. Centro de Atenção Psicossocial Infantil de São José dos Pinhais. Curitiba, PR, Brasil. (1)

${ }^{2}$ Enfermeira. Doutora em Enfermagem. Docente de Pós-Graduação em Enfermagem da Universidade Federal do Paraná. Curitiba, PR, Brasil. $\bigcirc$

${ }^{3}$ Discente de Enfermagem. Universidade Federal do Paraná. Curitiba, PR, Brasil. 9

${ }^{4}$ Enfermeira. Doutoranda em Enfermagem. Centro de Atenção Psicossocial Álcool e Drogas de Curitiba. Curitiba, PR, Brasil. ()

${ }^{5}$ Médico. Residente em Pediatria. Hospital Universitário do Oeste do Paraná. Cascavel, PR, Brasil. 


\title{
DIMENSIONS THAT INTERFERE WITH ADHERENCE TO MEDICATION IN SUBSTANCE-RELATED DISORDERS: INTEGRATIVE REVIEW
}

\begin{abstract}
Objective: To analyze the scientific production about the dimensions that interfere in the adherence to pharmacological therapy of individuals with mental disorders related to substance abuse from 2006 to April 2017.

Method: Integrative literature review of publications indexed in the following three databases LILACS, CINAHL and PUBMED, performed in April 2017.

Results: The 43 studies that met the eligibility criteria were analyzed. Such analysis made it possible to identify factors that have positive or negative impact on adherence to pharmacological therapy, grouped according to the five dimensions that determine the adherence proposed by the World Health Organization, namely: socioeconomic, health team/ system, disease, treatment, and individual/patient.

Conclusion: Although pharmacological therapy is crucial in the management of individuals with substance-related disorders, implementing the treatment is challenging because of the different interactive dimensions that directly impact the process of treatment adherence.
\end{abstract}

DESCRIPTORS: Adherence to medication; Patient cooperation; Pharmacological treatment; Substance-Related Disorders; Mental health.

\section{DIMENSIONEES QUE INLFUYEN EN LA ADHESIÓN A LA MEDICACIÓN EN LOS TRASTORNOS ASOCIADOS A SUSTANCIAS: REVISIÓN INTEGRATIVA}

\begin{abstract}
RESUMEN
Objetivo: Analizar la producción científica acerca de las dimensiones que influyen en la adhesión a la terapéutica medicamentosa de personas con trastornos mentales asociados al uso de sustancia, en el periodo de 2006 a abril de 2017.

Método: Revisión Integrativa de la Literatura a partir de tres bancos de datos, LILACS, CINAHL y PUBMED, hecha en abril de 2017.

Resultados: Se evaluaron 43 estudios que atendían a los criterios de elegibilidad. El análisis de los estudios posibilitó identificar factores que tienen impacto positivo o negativo en la adhesión a la terapéutica medicamentosa. Esos factores se organizaron de acuerdo con las cinco dimensiones determinantes para la adhesión que propuso la Organización Mundial de la Salud: socioeconómica, equipo/sistema de salud, enfermedad, tratamiento, e individuo/ paciente.

Conclusión: A pesar de que la terapéutica medicamentosa es imprescindible en la administración de la persona con trastorno asociado a substancias, muchas veces representa un desafío a causa de las distintas dimensiones interactivas que influencian directamente en el proceso de adhesión.
\end{abstract}

DESCRIPTORES: Adhesión a medicación; Cooperación del paciente; Tratamiento farmacológico; Trastornos asociados al Uso de Sustancias; Salud mental. 
Os transtornos relacionados às substâncias se configuram um fenômeno complexo, intimamente relacionado ao meio em que o indivíduo está inserido. É compreendido etimologicamente como multifatorial, sendo considerado na atualidade um dos mais graves problemas de saúde pública em decorrência do aumento de sua prevalênciaa ${ }^{(1-2)}$.

Estima-se que globalmente 250 milhões de pessoas usam Substâncias Psicoativas (SPA), o que corresponde aproximadamente a $5 \%$ da população com idades entre 16 e 64 anos, dessas, em torno de 29,5 milhões possuem transtornos relacionados às substâncias, no qual o consumo é prejudicial, e necessitam de tratamento ${ }^{(3-4)}$.

O tratamento contempla diversas modalidades terapêuticas, incluindo a medicamentosa. No entanto, esta terapêutica é marcada por diversos desafios, entre eles, a adesão(5). Sabe-se que uma parcela significativa de pessoas com doenças crônicas necessita de medicações para o tratamento. Entretanto, estima-se que apenas $50 \%$ cumpram o tratamento de maneira adequada. Esse quantitativo é menor quando se trata de países em desenvolvimento como o Brasil(6).

Em uma pesquisa nos Estados Unidos ${ }^{(1)}$ realizada por meio da análise de prontuários de pessoas acometidas pelos transtornos relacionados às substâncias em onze ambulatórios de diferentes cidades, observou-se que a terapêutica medicamentosa é um recurso importante no tratamento de pessoas com transtornos relacionados às substâncias. Entretanto, muitos indivíduos tiveram dificuldades de completar o plano medicamentoso proposto ${ }^{(1-2)}$.

Há muitos preditores que favorecem ou não a continuidade do tratamento medicamentoso, como a própria característica dos transtornos relacionados às substâncias marcada pela elevada gravidade, sucessivas recaídas, fissura (craving), longo tempo de tratamento, medicações excessivas, falta de apoio familiar, entre outros ${ }^{(1,7)}$.

Diante dos diferentes fatores que interferem na adesão, a Organização Mundial da Saúde apresenta a adesão a partir de um fenômeno multidimensional influenciado por meio da interação do indivíduo com cinco dimensões determinantes (socioeconômica, equipe/sistema de saúde, doença, tratamento e ao indivíduo/paciente), desmitificando a crença de que o paciente é único responsável pela continuidade do seu tratamento(6).

A partir deste contexto, entende-se que a adesão ao tratamento é um processo complexo, permeado pela subjetividade do sujeito, como ele se relaciona consigo mesmo, com o transtorno e com o tratamento. Pode ser compreendida por atitudes e comportamentos que são emergidos da experiência pessoal, da motivação e da expectativa.

Deste modo, considerando a necessidade de melhor compreender os fatores relacionados à adesão a terapêutica medicamentosa desta clientela, este trabalho teve o objetivo de analisar a produção científica acerca das dimensões que interferem na adesão à terapêutica medicamentosa de pessoas com transtornos mentais relacionados ao uso de substância.

\section{MÉTODO}

Trata-se de uma Revisão integrativa da literatura realizada no mês de abril de 2017, estruturada em seis etapas: (1) Seleção da hipótese ou questão para revisão; (2) Amostragem ou busca na literatura; (3) Representação e caracterização dos estudos e seus achados; (4) Análise dos achados; (5) Interpretação dos resultados e, (6) Relato da Revisão(8).

Foi utilizada a estratégia para a elaboração da questão norteadora e deste modo obteve-se a seguinte questão: Quais são as evidểncias científicas sobre os fatores associados a adesão e não adesão a terapêutica medicamentosa? 
A seleção dos estudos foi realizada por três revisores de forma independente e como critérios de inclusão buscou-se: estudos primários publicados na íntegra, que abordem a adesão e não adesão terapêutica medicamentosa de pessoas com transtornos relacionados a substância, no período de 2006 a abril de 2017, publicados nos idiomas inglês, espanhol e português. Os critérios de exclusão restringiram estudos de revisão, de reflexão, metanálise, carta, editorial, estudo de caso e estudos que trazem como tema transtornos relacionados ao uso de tabaco.

A estratégia de busca foi estruturada a partir de descritores controlados indexados no Medical Subject Headings (MeSH) e no Descritores em Ciências da Saúde (Decs): Substance-Related Disorder; Alcohol-Related Disorders; Alcoholism; Amphetamine-Related Disorders; Cocaine-Related Disorders; Inhalant Abuse; Marijuana Abuse; Opioid-Related Disorders; Heroin Dependence; Morphine Dependence; Phencyclidine Abuse; Substance Abuse, Intravenous; Substance Abuse Detection e implementada nas bases de dados Literatura Latino Americana e do Caribe em Ciências da Saúde (LILACS), Cumulattive Index to Nursingand Alied Health Literature (CINAHL) e PUBMED, desenvolvida pela National Center for Biotechnology Information (NCBI) da US National Library of Medicine (NLM) considerando seus respectivos recursos de pesquisa.

A busca nos bancos de dados resultou em um total de 1.304 estudos, desses foram excluídos 1.209 após a leitura flutuante dos títulos e resumos permanecendo 319. Após a leitura na íntegra, foram excluídos mais 276 , compondo a amostra final um total de 43 estudos.

Os dados coletados foram analisados quantitativamente de maneira descritiva e agrupadas de acordo as cinco dimensões determinantes para a adesão proposta pela Organização Mundial da Saúde: socioeconômico, equipe/sistema de saúde, doença, tratamento e indivíduo/paciente.

Este artigo originou-se do projeto de pesquisa intitulado 'Adesão ao tratamento pela pessoa com transtornos relacionados ao uso de substâncias psicoativas' aprovado Comitê de Ética em Pesquisa do Setor de Ciências da Saúde da Universidade Federal do Paraná, sob número do parecer 2.033.006.

\section{RESULTADOS}

Foram obtidos um estudo na base de dados LILACS, 15 na PUBMED e 27 na CINAHAL. $O$ inglês foi o idioma encontrado em todos os estudos. O número de autores variou de um a 13, e devido ao elevado número de autores, optou-se por caracterizar somente o primeiro autor sendo a maioria da área médica (29), apenas um estudo foi realizado por enfermeiro.

No que se refere à instituição na qual os estudos foram desenvolvidos, 35 foram oriundos de universidade, sete de centros de pesquisa e somente um de hospital. Quanto aos periódicos, 36 eram multiprofissionais destinados à temática sobre substâncias psicoativas, e considerando o país de origem, 27 foram realizados nos Estados Unidos da América, seis na China e um no Brasil.

O diagnóstico médico prevalente foi dependência por opioides caracterizado em 25 estudos, seguido de dependência por álcool descrito em 12 estudos. Quanto aos critérios metodológicos, observou-se baixo nível de evidência entre os estudos, com 25 apresentando nível de evidência igual a 6, e 14 nível de evidência 2.

Os fatores que interferem na adesão e na não adesão a terapêutica medicamentosa encontrados nos estudos foram classificados de acordo com as cinco diferentes dimensões proposta pela $\mathrm{OMS}^{(6)}$ e apresentados no Quadro 1. 
Quadro 1 - Dimensões interativas que interferem a adesão. Curitiba, PR, Brasil, 2017 (continua)

\begin{tabular}{|c|c|c|}
\hline \multicolumn{3}{|c|}{ Dimensão Tratamento } \\
\hline \multirow{10}{*}{$\begin{array}{l}\text { Fatores } \\
\text { de } \\
\text { Adesão }\end{array}$} & & $\mathrm{n}$ \\
\hline & 1. Outras modalidades terapêuticas ${ }^{(1,5,9-34)}$ & 28 \\
\hline & 2. Dose adequada da medicação(10-12,24,26,28,31,35-37,39) & 12 \\
\hline & 3. Eficácia da medicação(5,9,35) & 3 \\
\hline & 4. Tratamentos prévios ${ }^{(14,21,24)}$ & 3 \\
\hline & 5. Medicação injetável(37) & 1 \\
\hline & 6. Sem histórico de tratamento(36) & 1 \\
\hline & 7. Tempo longo de tratamento(24) & 1 \\
\hline & 8. Triagem toxicológica ${ }^{(25)}$ & 1 \\
\hline & TOTAL & 50 \\
\hline \multirow{9}{*}{$\begin{array}{l}\text { Fatores } \\
\text { de Não } \\
\text { Adesão }\end{array}$} & 1. Efeitos colaterais e adversos da medicação ${ }^{(12,15,18,20,22,26,32,40,41)}$ & 9 \\
\hline & 2. Longo tempo de tratamento medicamentoso $(11,15,33,36,38,42-45)$ & 9 \\
\hline & 3. Baixa eficácia da medicação $o^{(12,18,27,35,37-38)}$ & 6 \\
\hline & 4. Uso de medicações para outras comorbidades ${ }^{(9,37,44)}$ & 3 \\
\hline & 5. Longo tempo entre as doses inicial e de estabilização(24,26) & 2 \\
\hline & 6. Alteração da dosagem da medicação sem orientação médica ${ }^{(43)}$ & 1 \\
\hline & 7. Histórico de tratamentos anteriores ${ }^{(13)}$ & 1 \\
\hline & 8. Dificuldade de comparecer em decorrência do trabalho(46) & 1 \\
\hline & TOTAL & 32 \\
\hline \multicolumn{3}{|c|}{ Dimensão Doença } \\
\hline \multirow{7}{*}{$\begin{array}{l}\text { Fatores } \\
\text { de } \\
\text { Adesão }\end{array}$} & 1. Abstinência da substância de preferência ${ }^{(10,15,18,24,28)}$ & 6 \\
\hline & 2. Menor gravidade da dependência química ${ }^{(20,30,39)}$ & 3 \\
\hline & 3. Comorbidades mentais de baixa gravidade $(17,25,40)$ & 3 \\
\hline & 4. Uso de outras substâncias psicoativas ${ }^{(38,47)}$ & 2 \\
\hline & 5. Recaída ${ }^{(12-31)}$ & 2 \\
\hline & 6. Comorbidade clínica(48) & 1 \\
\hline & TOTAL & 17 \\
\hline \multirow{8}{*}{$\begin{array}{l}\text { Fatores } \\
\text { de Não } \\
\text { Adesão }\end{array}$} & 1. Não se manter abstinente $e^{(5,9-10,13-14,17,19-24,28,33-35,39,49)}$ & 19 \\
\hline & 2. Comorbidades mentais associadas $(1,5,9,13,17,26,28,33,35,37,44-45,48-50)$ & 15 \\
\hline & 3. Gravidade da dependência química ${ }^{(10,16,30,32-33,36,44,49-50)}$ & 9 \\
\hline & 4. Uso de múltiplas drogas ${ }^{(17-18,26,28,46)}$ & 6 \\
\hline & 5. Tipo de substância (heroína e cocaína) ${ }^{(15,42,48)}$ & 3 \\
\hline & 6. Síndrome da abstinência ${ }^{(26,39)}$ & 2 \\
\hline & 7. Fissura não controlada ${ }^{(12)}$ & 1 \\
\hline & TOTAL & 55 \\
\hline
\end{tabular}


Quadro 1 - Dimensões interativas que interferem a adesão. Curitiba, PR, Brasil, 2017 (continuação)

\begin{tabular}{|c|c|c|}
\hline \multicolumn{3}{|c|}{ Dimensão Socioeconômica } \\
\hline \multirow{12}{*}{$\begin{array}{l}\text { Fatores } \\
\text { de } \\
\text { Adesão }\end{array}$} & 1. Idade: Mais elevada(13-14,17,20-23,26,33,35,42) & 13 \\
\hline & Menos elevada(19) & 1 \\
\hline & 2. Situação empregatícia: Empregado(13,26,46) & 3 \\
\hline & Aposentado(13) & 1 \\
\hline & 3. Maiores níveis escolares ${ }^{(13,44)}$ & 2 \\
\hline & 4. Sexo feminino $(19,26)$ & 2 \\
\hline & 5. Estado conjugal: Solteiro(19) & 1 \\
\hline & Casado(44) & 1 \\
\hline & 6. Situação Financeira: Estável(44) & 1 \\
\hline & Elevada $^{(13)}$ & 1 \\
\hline & 7. Ambiente familiar favorável(48) & 1 \\
\hline & TOTAL & 27 \\
\hline \multirow{15}{*}{$\begin{array}{l}\text { Fatores } \\
\text { de Não } \\
\text { Adesão }\end{array}$} & 1. Idade menos avançada $(22-23,26,28,44,49)$ & 6 \\
\hline & 2. Ter infrações penais ${ }^{(16,23,26,48)}$ & 4 \\
\hline & 3. Alto custo do tratamento $(25,30,45)$ & 3 \\
\hline & 4. Etnia negra $(26,48)$ & 2 \\
\hline & 5. Sexo masculino $(16,26)$ & 2 \\
\hline & 6. Desemprego ${ }^{(15-44)}$ & 2 \\
\hline & 7. Prejuízo no trabalho(44) & 1 \\
\hline & 8. Situação de rua(15) & 1 \\
\hline & 9. Baixa renda ${ }^{(15)}$ & 1 \\
\hline & 10. Estado civil solteiro(15) & 1 \\
\hline & 11. Baixa escolaridade ${ }^{(44)}$ & 1 \\
\hline & 12. Ter filhos ${ }^{(10)}$ & 1 \\
\hline & 13. Situação de prisão(5) & 1 \\
\hline & 14. Falta de incentivo financeiro para a adesão(50) & 1 \\
\hline & TOTAL & 27 \\
\hline \multicolumn{3}{|c|}{ Dimensão Indivíduo/Paciente } \\
\hline \multirow{7}{*}{$\begin{array}{l}\text { Fatores } \\
\text { de } \\
\text { Adesão }\end{array}$} & 1. Desejo de cessar o uso da SPA $(9,16)$ & 2 \\
\hline & 2. Aceitar a terapêutica medicamentosa ${ }^{(9,11)}$ & 2 \\
\hline & 3. Satisfação com o tratamento medicamentoso ${ }^{(16,37)}$ & 2 \\
\hline & 4. Satisfação com o tratamento psicossocial|(20,25) & 2 \\
\hline & 5. Compreender a importância da terapêutica medicamentosa ${ }^{(9,38)}$ & 2 \\
\hline & 6. Trauma emocional|(19) & 1 \\
\hline & TOTAL & 11 \\
\hline
\end{tabular}


Quadro 1 - Dimensões interativas que interferem a adesão. Curitiba, PR, Brasil, 2017 (continuação)

\begin{tabular}{|c|c|c|}
\hline \multirow{14}{*}{$\begin{array}{l}\text { Fatores } \\
\text { de Não } \\
\text { Adesão }\end{array}$} & 1. Insatisfação com o profissional(20,27-28,33,49) & 5 \\
\hline & 2. Baixo entendimento sobre a importância da medicação ${ }^{(16,20,22,35)}$ & 4 \\
\hline & 3. Desejo de interromper o tratamento medicamentoso ${ }^{(14,22,33)}$ & 3 \\
\hline & 4. Desorganização(5,44) & 2 \\
\hline & 5. Desmotivação quanto ao tratamento medicamentoso ${ }^{(22)}$ & 1 \\
\hline & 6. Dificuldade de autoadministração de medicações injetáveis ${ }^{(37)}$ & 1 \\
\hline & 7. Ausência de desejo em tomar a medicação regularmente ${ }^{(33)}$ & 1 \\
\hline & 8. Expectativa de curto tempo de tratamento medicamentoso ${ }^{(36)}$ & 1 \\
\hline & 9. Baixo comprometimento com o tratamento medicamentoso ${ }^{(45)}$ & 1 \\
\hline & 10. Experiências negativas com tratamentos anteriores ${ }^{(17)}$ & 1 \\
\hline & 11. Esquecimento ${ }^{(33)}$ & 1 \\
\hline & 12. Falta de conhecimento sobre a medicação/dose/horários ${ }^{(40)}$ & 1 \\
\hline & 14. Impulsividade(5) & 1 \\
\hline & TOTAL & 23 \\
\hline \multicolumn{3}{|c|}{ Dimensão Equipe/Sistema de saúde } \\
\hline \multirow{4}{*}{$\begin{array}{l}\text { Fatores } \\
\text { de } \\
\text { Adesão }\end{array}$} & 1. Assistência pública aos medicamentos ${ }^{(48)}$ & 1 \\
\hline & 2. Organização adequada do ambiente de tratamento ${ }^{(42)}$ & 1 \\
\hline & 3. Seguro saúde de melhor qualidade ${ }^{(47)}$ & 1 \\
\hline & TOTAL & 3 \\
\hline \multirow{5}{*}{$\begin{array}{l}\text { Fatores } \\
\text { de Não } \\
\text { Adesão }\end{array}$} & 1. Acesso limitado aos locais de tratamento $(5,25,33)$ & 3 \\
\hline & 2. Falta de comunicação com os profissionais $(5,27,49)$ & 3 \\
\hline & 3. Horário de tratamento limitado(5) & 1 \\
\hline & 4. Falta de apoio profissional|(37) & 1 \\
\hline & TOTAL & 8 \\
\hline
\end{tabular}

\section{DISCUSSÃO}

Evidenciou-se a predominância de estudos publicados por pesquisadores norteamericanos em comparação a de brasileiros. Isso demonstra a importante lacuna em pesquisas acerca da adesão e não adesão a terapêutica medicamentosa de pessoas com transtornos relacionados às substâncias no Brasil, que reflete na adesão a este recurso terapêutico e pode ser um indicativo do sucesso do tratamento como um todo(26).

Outro aspecto de destaque refere-se ao quantitativo expressivo de estudos desenvolvidos por médicos, quando o fenômeno da adesão e não adesão a terapêutica medicamentosa deve ser interesse de todas as profissões envolvidas com o tratamento e cuidado a pessoas com transtornos relacionados às substâncias. Não obstante, é imprescindível para o gerenciamento do transtorno, porque propicia a minimização e ou a remissão dos sinais e sintomas da abstinência e da fissura, auxilia na reduçã̃o de morbidades, favorece a motivação para a reabilitação, na prevenção de lapsos e recaídas, e contribui com a reinserção social(7). 
de adesão mais encontrado 'seguir corretamente a prescrição'. Contudo, essa definição contraria a da Organização Mundial da Saúde, para a qual a adesão a um tratamento é definida pelo grau em que o comportamento da pessoa ao tomar uma medicação, seguir um regime alimentar e ou executar mudanças no estilo de vida corresponde às recomendações acordadas com um prestador de cuidados de saúde ${ }^{(6)}$.

A terapêutica medicamentosa é um recurso importante no tratamento dos transtornos relacionados a substâncias, entretanto a adesão pode ser um desafio, uma vez que muitos indivíduos que sofrem deste transtorno mental têm dificuldade de completar o tratamento necessário em decorrência de diversos fatores ${ }^{(1,51-52)}$.

Quanto aos fatores relacionados à adesão e não adesão na dimensão Tratamento, obteve-se destaque para a participação em outras modalidades terapêuticas, a dose adequada da medicação, efeitos colaterais e o longo tempo de tratamento, identificados como fatores que influenciam a adesão. Diante da complexidade de fenômenos envolvidos e a ampla diversidade de fatores que impactam os transtornos relacionados a substâncias, sabe-se que aliar diferentes modalidades terapêuticas à medicamentosa favorece a efetividade do tratamento e estimula o desenvolvimento de atitudes positivas do indivíduo, refletindo na prevenção da recaída e na manutenção da abstinência ${ }^{(5)}$.

Destarte, na análise dos estudos da revisão integrativa evidenciou-se a necessidade de um tratamento multidisciplinar, a partir de abordagens psicossociais que contemplem o indivíduo, a família e a sociedade, que promova a qualidade de vida, fortaleça a autonomia e a reinserção social ${ }^{(7,51)}$.

Indiscutivelmente o uso contínuo e correto da medicação está diretamente relacionado à eficácia e a redução da morbimortalidade, contudo a prescrição da medicação deve ser realizada de modo correspondente aos requisitos individuais, durante o período de tempo de tratamento necessário e com menor custo possível, ${ }^{(51-52)}$ o que, por sua vez, pode atenuar o comportamento de não adesão ${ }^{(51-52)}$.

Apesar da importância da adesão no processo de reabilitação dos transtornos relacionados às substâncias, evidencia-se elevados índices de abandono da medicação, principalmente quando o indivíduo se depara com o extenso período de tempo de tratamento e com os inúmeros efeitos colaterais e adversos que se tornam constante desafio para a continuidade do tratamento ${ }^{(1-2)}$.

Aqueles que são acometidos pelos transtornos relacionados às substâncias enfrentam as consequências de um processo de tratamento longo e contínuo, marcado por alterações biológicas e psicológicas que podem persistir mesmo quando ocorre a desintoxicação, ou que levam a efeitos comportamentais que se manifestam em forma de constantes recaídas e no craving intenso, principalmente quando expostos a estímulos relacionados à substância ${ }^{(53)}$.

A Doença é outra dimensão relacionada à adesão a terapêutica medicamentosa, a qual emergiram a abstinência, comorbidades mentais, a gravidade dos transtornos relacionados a substâncias e o uso de múltiplas drogas, como fatores de maior destaque nos estudos.

Sabe-se que manter-se abstinente auxilia na adesão ao tratamento, pois este comportamento demonstra que o indivíduo está fortalecido e consciente de seu problema e disposto a engajar-se em ações específicas que visem a manutenção da abstinência ${ }^{(7)}$. Nesse caso a terapêutica medicamentosa configura-se como um recurso que auxilia nesse processo, pois busca restaurar o equilíbrio das funções psíquicas e consequentemente o comportamento controlando os sintomas agudos, favorecendo a continuidade do tratamento ${ }^{(51,53)}$.

A comorbidade mental foi destacada nos estudos da revisão integrativa como um fator que interfere na adesão a terapêutica medicamentosa. Sabe-se que associação com outras comorbidades mentais intensifica o sofrimento psíquico do indivíduo, o que pode 
representar um desafio na manutenção da abstinência.

Outro fator pertencente à dimensão Doença com destaque na revisão integrativa foi a gravidade dos transtornos relacionados a substâncias. De acordo com o Diagnostic and Statistical Manual of Mental Disorders em sua quinta edição (DSM V), a gravidade destes transtornos é classificada em leve, moderada e grave. A diferença entres essas classes resulta no agrupamento de diferentes critérios pré-estabelecidos fundamentados em um padrão patológico de comportamento relacionado ao uso. Neste sentido, quanto maior a gravidade do transtorno, maior a negligência que o indivíduo irá apresentar para cumprir com suas atividades, entre elas o tratamento.

O tipo de substância psicoativa de preferência também foi referenciado pelos autores dos estudos da revisão integrativa, como fator capaz de impactar a adesão ao tratamento, ao que indivíduos que consomem heroína ou cocaína/crack apresentam menores níveis de adesão ao tratamento medicamentoso. Estas substâncias caracterizam-se por seu alto potencial aditivo decorrente da intensa fissura provocada pelo uso, resultando em um consumo repetitivo e exacerbado, assim a dependência dessas substâncias se estabelece de forma mais rápida(7,53).

Desta mesma forma, destaca-se que pessoas que fazem uso de mais de um tipo de substância psicoativa apresentam dificuldades em seguir adequadamente a terapêutica medicamentosa, ${ }^{(7)}$ o consumo de uma maior quantidade de drogas resulta em maiores danos ao indivíduo, pois há a possibilidade de este desenvolver a dependência em mais de uma substância. Além disto, o uso de múltiplas drogas compromete o tratamento, pois pode resultar na interação entre as substâncias utilizadas, ocasionando efeitos clínicos e comportamentais adversos que dificultam a adesão(7,51).

Na dimensão sociodemográfica, destacam-se para a idade, situação empregatícia, escolaridade, sexo e infrações penais. Alguns estudos apontaram que indivíduos do sexo masculino com idade menos elevada estão mais suscetíveis a não aderir ao tratamento medicamentoso ${ }^{(31)}$.

Dados de um estudo retrospectivo realizado em um hospital psiquiátrico situado em Minas Gerais, Brasil, reforçam este achado em que a partir da coleta de informações em bancos de dados, os autores identificaram a prevalência de $4.325(67,78 \%)$ indivíduos do sexo masculino admitidos no serviço, desde a inauguração da clínica em 1980 até o ano de $2005^{(54)}$.

Em contrapartida, alguns autores sugerem que a prevalência de transtornos relacionados às substâncias em mulheres pode ter sido ou estar sendo subnotificado, sendo apontada como uma possível causa, o fato de que as mulheres buscam tratamento com menos frequência em decorrência de sentimentos de vergonha, medo de julgamento e ou preconceito, além de este aspecto poder estar associado ao estilo de vida e ao comportamento de isolamento social|(55).

Assim, embora a literatura aponte um percentual reduzido de mulheres que fazem o uso abusivo de substâncias psicoativas, faz-se necessário investigar com maior aprofundamento esta população, a fim de identificar as suas particularidades e poder contribuir para a elaboração de um cuidado e tratamento mais efetivos.

Já a baixa escolaridade é referida nos estudos como um fator para a não adesão, aspecto que pode ser justificado pelo início do consumo da substância psicoativa ocorrer ainda na adolescência, enquanto o indivíduo está em processo de aprendizagem e ou escolarização. Como consequência, abandonam os estudos, uma vez que passam a apresentar mudanças no comportamento e dificuldades no relacionamento interpessoal, evidenciados por condutas inadequadas, além de ter a cognição prejudicada em decorrência do uso contínuo da substância( ${ }^{(5)}$.

Observou-se em geral que maiores níveis de escolaridade favorecem a adesão ao tratamento, ao que se pode atribuir que este facilita o entendimento dos aspectos 
relacionados a terapêutica medicamentosa, bem como a necessidade e a importância do correto seguimento ${ }^{(16)}$.

Ter histórico de infrações penais foi observado como um fator para a não adesão ao tratamento. Compreende-se que o forte desejo por consumir a substância psicoativa, associado ao alto custo de manutenção da dependência, faz com que o indivíduo muitas vezes apresente comportamentos de risco, sendo capaz de cometer infrações e delitos, tal como tráfico, roubos ou furtos, para a obtenção da substância de preferểncia ${ }^{(41)}$.

Na dimensão Indivíduo/Paciente ressalta-se que o não entendimento das informações fornecidas sobre a terapêutica medicamentosa pode trazer diversas consequências negativas, ocasionando o insucesso do tratamento, como o atraso na administração do medicamento, agravando o transtorno mental do indivíduo, aumento dos efeitos colaterais, erros no esquema de medicação, entre outros, todos esses fatores favorecem a não adesão, o que impossibilita a busca de um tratamento efetivo e seguro(56).

A adesão ao tratamento medicamentoso é um processo multicausal que envolve o indivíduo em sua totalidade e o contexto sociocultural no qual está inserido, assim é essencial identificar as particularidades e o perfil das pessoas que fazem o uso abusivo de substâncias psicoativas, a fim de conhecer os determinantes para a adesão ou não adesão ao tratamento ${ }^{(6)}$.

$\mathrm{Na}$ dimensão Equipe/Sistema de saúde, houve menor número de fatores: foram encontrados acesso limitado aos locais de tratamento e falta de comunicação com os profissionais. Quanto ao primeiro fator, destaca-se que as estratégias de tratamento devem ser pautadas na reabilitação social e na redução de danos, centrada principalmente em ações comunitárias, integrando políticas públiças que visam, de forma intersetorial, favorecer a ampliação do cuidado por meio de estratégias que aproximem o indivíduo e a família ao local de tratamento(57).

Ressalta-se que o atendimento a essa clientela também deve ocorrer no âmbito da atenção básica, tornando possível salvaguardar a conquista do Movimento da Reforma Psiquiátrica da descentralização da saúde mental. Portanto, estratégias voltadas para o trabalho em rede com apoio matricial da atenção especializada se fazem necessárias ${ }^{(57)}$.

Já em relação à comunicação com a equipe de saúde, entende-se que ouvir e conhecer o indivíduo são as mais apropriadas alternativas para a melhor condução do tratamento. A comunicação adequada entre o paciente e o profissional possibilita resultados mais eficientes e menos onerosos, pois permite que o indivíduo opine e participe de seu próprio tratamento, fortalecendo seu protagonismo ${ }^{(58)}$.

Atuar na saúde mental é uma tarefa árdua, requer do profissional um envolvimento com o usuário com o intuito de responsabilizar-se e solidarizar-se com o sofrimento do outro. Portanto, a vinculação deve ser iniciada por meio da disponibilidade para a escuta, empatia, comprometimento e a comunicação com o indivíduo ${ }^{(54)}$. Entende-se que esse modo de trabalho permite a construção de uma aliança terapêutica fortalecida entre o indivíduo que necessita de tratamento e a equipe de saúde. $E$ esse fato, adicionado à segurança $e$ credibilidade do profissional, favorece a adesão a terapêutica medicamentosa ${ }^{(58)}$.

\section{CONCLUSÃO}

Diante da complexidade das diferentes dimensões que envolvem a adesão ao tratamento medicamentoso, esta Revisão Integrativa possibilitou a apresentação de dados relevantes acerca dos fatores que podem subsidiar estratégias de cuidado a pessoas com transtornos relacionados a substância.

Neste contexto, o profissional da enfermagem, como integrante da equipe multiprofissional, deve desenvolver ações para a adesão a terapêutica medicamentosa, a 
fim de conscientizar o indivíduo quanto às situações de alto risco para a recaída, auxiliálo no processo de enfrentamento e de mudança de hábitos, proporcionando melhora da qualidade de vida, além de auxiliar na manutenção da abstinência, bem como na reabilitação e reinserção social.

Destaca-se o baixo quantitativo de publicações brasileiras acerca desta temática, porquanto, é necessário incentivar as publicações nacionais, haja visto que por meio de publicações em periódicos científicos ocorre a comunicação dos resultados de pesquisa, contribuindo com o avanço da ciência, propiciando transformações no conhecimento que assegura e subsidia a prática profissional.

\section{REFERÊNCIAS}

1. Stout RL, Braciszewski JM, Subbaraman MS, Kranzler HR, O'Malley SS, Falk D. What happens when people discontinue taking medications? Lessons from COMBINE. Addiction [Internet]. 2014 [acesso em 25 set 2017]; 109(12). Disponível em: https://doi.org/10.1111/add.12700.

2. Alvarez PES, Rosendo E, Alchieri JC. The applicability of the concept of treatment adherence in the context of the Brazilian mental health system. Rev. Esc. Enferm. USP. [Internet.] 2016 [acesso em 25 set 2017]; 50(Espec). Disponível em: http://dx.doi.org/10.1590/S0080-623420160000300008.

3. World Health Organization (WHO). Health topics: Substance abuse. WHO [Internet]. 2017 [acesso em 29 ago 2017]. Disponível em: http://www.emro.who.int/health-topics/substance-abuse/index.html.

4. United Nations Office on Drugs and Crime (UNODC). World Drug Report 2017 [Internet]. Vienna: United Nations Office on Drugs and Crime; 2017.

5. Zhang B, Cai T, Yan Z, Mburu G, Wang B, Yang L. Impact of blended treatment literacy and psychoeducation on methadone maintenance treatment outcomes in Yunnan, China. Harm Reduct J [Internet]. 2016 [acesso em 22 fev 2017]; 13(8). Disponível em: https://doi.org/10.1186/s12954-0160097-y.

6. World Health Organization (WHO). Adherence to long-term therapies: Evidence for action. Geneva: World Health Organization; 2003.

7. Ferreira ACZ, Czarnobay J, Borba LO, Capistrano FC, Kalinke LP, Maftum MA. Determinantes intra e interpessoais da recaída de dependentes químicos. Rev. Eletr. Enf. [Internet]. 2016 [acesso em 09 ago 2017]; 18(e1114). Disponível em: https://dx.doi.org/10.5216/ree.v18.34292.

8. Ganong LH. Integrative reviews of nursing research. Res. Nurs. Health [Internet]. 1987 [acesso em 09 ago 2017]; 10(1). Disponível em: https://doi.org/10.1002/nur.4770100103.

9. Finnell DS, Osborne FH. Stages of change for psychotropic medication adherence and substance cessation. Arch Psychiatr Nurs [Internet]. 2006 [acesso em 09 ago 2017]; 20(4). Disponível em: https://doi. org/10.1016/j.apnu.2005.12.006.

10. Peles E, Schreiber S, Adelson M. Factors predicting retention in treatment: 10-year experience of methadone maintenance treatment (MMT) clinic in Israel. Drug Alcohol Depend [Internet]. 2006 [acesso em 09 ago 2017]; 82(3). Disponível em: https://doi.org/10.1016/j.drugalcdep.2005.09.004.

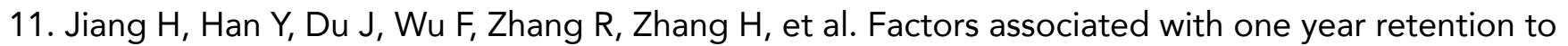
methadone maintenance treatment program among patients with heroin dependence in China. Subst Abuse Treat Prev Policy [Internet]. 2014 [acesso em 15 ago 2017]; 9(11). Disponível em: https://doi. org/10.1186/1747-597X-9-11.

12. Baros AM, Latham PK, Moak DH, Voronin K, Anton RF. What role does measuring medication compliance play in evaluating the efficacy of naltrexone? Alcohol Clin Exp Res [Internet]. 2007 [acesso em 12 ago 2017]; 31(4). Disponível em: https://doi.org/10.1111/j.1530-0277.2007.00343.x. 
13. Oslin DW, Lynch KG, Pettinati HM, Kampman KM, Gariti P, Gelfand L, et al. A placebo-controlled randomized clinical trial of naltrexone in the context of different levels of psychosocial intervention. Alcohol Clin Exp Res [Internet]. 2008 [acesso em 12 ago 2017]; 32(7). Disponível em: https://doi. org/10.1111/j.1530-0277.2008.00698.x.

14. Kranzler HR, Stephenson JJ, Montejano L, Wang S, Gastfriend DR. Persistence with oral naltrexone for alcohol treatment: implications for healthcare utilization. Addiction [Internet]. 2008 [acesso em 11 ago 2017]; 103(11). Disponível em: https://dx.doi.org/10.1111/j.1360-0443.2008.02345.x.

15. Banta-Green CJ, Maynard C, Koepsell TD, Wells EA, Donovan DM. Retention in methadone maintenance drug treatment for prescription-type opioid primary users compared to heroin users. Addicion. [Internet]. 2009 [acesso em 15 ago 2017]; 104(5). Disponível em: https://dx.doi.org/10.1111/ j.1360-0443.2009.02538.x.

16. Nuijten M, Blanken P, Van den Brink W, Hendriks V. Treatment of crack-cocaine dependence with topiramate: a randomized controlled feasibility trial in The Netherlands. Drug Alcohol Depend. [Internet]. 2014 [acesso em 10 ago 2017]; 138. Disponível em: https://dx.doi.org/10.1016/j.drugalcdep.2014.02.024.

17. Streel E, Chenut C, Papageorgiou C, Verbanck P. DMS IV axis II traits can influence compliance to treatment with oral naltrexone: a preliminary study on 30 opiate dependent patients. Addict Behav. [Internet]. 2014 [acesso em 05 set 2017]; 39(1). Disponível em: https://dx.doi.org/10.1016/j. addbeh.2013.08.031.

18. Kelly SM, O'Grady KE, Mitchell SG, Brown BS, Schwartz RP. Predictors of methadone treatment retention from a multi-site study: a survival analysis. Drug Alcohol Depend. [Internet]. 2011 [acesso em 22 set 2017]; 117(2-3). Disponível em: https://dx.doi.org/10.1016/j.drugalcdep.2011.01.008.

19. Gu J, Xu H, Lau JTF, Hao Y, Zhong Y, Fan L, et al. Misconceptions predict dropout and poor adherence prospectively among newly admitted first-time methadone maintenance treatment clients in Guangzhou. Addiction [Internet]. 2012 [acesso em 20 set 2017]; 107(9). Disponível em: https://dx.doi.org/10.1111/ j.1360-0443.2012.03859.x.

20. Peters EN, Leeman RF, Fucito LM, Toll BA, Corbin WR, O'Malley SS. Co-occurring marijuana use is associated with medication nonadherence and nonplanning impulsivity in young. Addict Behav. [Internet]. 2012 [acesso em 27 set 2017]; 37(4). Disponível em: https://dx.doi.org/10.1016/j.addbeh.2011.11.036.

21. Baxter JD, Clark RE, Samnaliev M, Aweh G, O'Connell E. Adherence to Buprenorphine Treatment Guidelines in a Medicaid Program. Subst. Abus. [Internet]. 2015 [acesso em 15 set 2017]; 36(2). Disponível em: https://dx.doi.org/10.1080/08897077.2014.991469.

22. DeFulio A, Everly JJ, Leoutsakos JMS, Umbricht A, Fingerhood M, Bigelow GE, et al. Employmentbased reinforcement of adherence to an FDA approved extended release formulation of naltrexone in opioid-dependent adults: A randomized controlled trial. Drug Alcohol Depend. [Internet]. 2012 [acesso em 12 set 2017]; 120(1-3). Disponível em: https://dx.doi.org/10.1016/j.drugalcdep.2011.06.023.

23. Gueorguieva R, Wu R, Krystal JH, Donovan D, O'Malley SS. Temporal patterns of adherence to medications and behavioral treatment and their relationship to patient characteristics and treatment response. Addict Behav. [Internet]. 2013 [acesso em 05 out 2017]; 38(5). Disponível em: https://dx.doi. org/10.1016/j.addbeh.2013.01.024.

24. Walcher S, Koc J, Reichel V, Schlote F, Verthein U, Reimer J. The opiate dosage adequacy scale for identification of the right methadone dose-a prospective cohort study. BMC Pharmacol Toxicol [Internet] 2016;17(15) [acesso em 10 out 2017]. Disponível: https://dx.doi.org/10.1186/s40360-016-0058-9.

25. Clark CB, Hendricks PS, Lane PS, Trent L. Cropsey KL. Methadone maintenance treatment may improve completion rates and delay opioid relapse for opioid dependent individuals under community corrections supervision. Addict Behav. [Internet]. 2014 [acesso em 23 ago 2017]; 39(12). Disponível em: https://dx.doi.org/10.1016/j.addbeh.2014.07.011.

26. Bukten A, Skurtveit S, Waal H, Clausen T. Factors associated with dropout among patients in opioid maintenance treatment (OMT) and predictors of re-entry. A national registry-based study. Addict 
Behav. [Internet]. 2014 [acesso em 20 out 2017]; 39(10). Disponível em: https://dx.doi.org/10.1016/j. addbeh.2014.05.007.

27. Launoen E, Wallace I, Kotovirta E, Alho H, Simojoki K. Factors associated with non-adherence and misuse of opioid maintenance treatment medications and intoxicating drugs among Finnish maintenance treatment patients. Drug Alcohol Depend. [Internet]. 2016 [acesso em 16 dez 2017]; 162. Disponível em: https://dx.doi.org/10.1016/j.drugalcdep.2016.03.017.

28. McRae-Clark AL, Baker NL, Sonne SC, DeVane CL, Wagner A, Norton J. Concordance of direct and indirect measures of medication adherence in a treatment trial for cannabis dependence. J Subst abuse treat [Internet]. 2015 [acesso em 26 nov 2017]; 57. Disponível em: https://dx.doi.org./10.1016/j. jsat.2015.05.002.

29. Stoner SA, Arenella PB, Hendershot CS. Randomized controlled trial of a mobile phone intervention for improving adherence to naltrexone for alcohol use disorders. PLoS One [Internet]. 2015 [acesso em 21 set 2017]; 10(4). Disponível em: https://dx.doi.org/10.1371/journal.pone.0124613.

30. Sullivan MA, Bisaga A, Glass A, Mishlen K, Pavlicova M, Carpenter KM, et al. Opioid use and dropout in patients receiving oral naltrexone with or without single administration of injection naltrexone.

Drug Alcohol Depend. [Internet]. 2015 [acesso em 18 dez 2017]; (147). Disponível em: https://dx.doi. org/10.1016/j.drugalcdep.2014.11.028.

31. Heffner JL, Tran GQ, Johnson CS, Barret SW, Blom TJ, Thompson RD, et al. Combining motivational interviewing with compliance enhancement therapy (MI-CET): development and preliminary evaluation of a new, manual-guided psychosocial adjunct to alcohol-dependence pharmacotherapy. J Stud Alcohol Drugs [Internet]. 2010 [acesso em 14 set 2017]; 71(1). Disponível em: https://dx.doi.org/10.15288/ jsad.2010.71.61.

32. Stoner SA, Hendershot CS. A randomized trial evaluating an mHealth system to monitor and enhance adherence to pharmacotherapy for alcohol user disorders. Addict Sci Clin Pract [Internet]. 2012 [acesso em 9 ago 2017]; 7(9). Disponível em: https://dx.doi.org/10.1186/1940-0640-7-9.

33. Marienfeld C, Liu P, Wang X, Schottenfeld R, Zhou W, Chawarski MC. Evaluation of an implementation of methadone maintenance treatment in China. Drug Alcohol Depend. [Internet]. 2015 [acesso em 06 set 2017]; 157. Disponível em: https://dx.doi.org/10.1016/j.drugalcdep.2015.10.001.

34. Melnyk B, Fineout-Overholt E. Evidence-Based Practice in Nursing \& Healthcare: a guide to best practice. 2nd ed. China: Lippincott Williams \& Wilkins; 2011.

35. Holt M. Agency and dependency within treatment: drug treatment clients negotiating methadone and antidepressants. Soc Sci Med [Internet]. 2007 [acesso em 09 ago 2017]; 64(9). Disponível em: https://doi. org/10.1016/j.socscimed.2007.01.011.

36. Zweben A, Pettinati HM, Weiss RD, Youngblood M, Cox CE, Mattson ME, et al. Relationship between medication adherence and treatment outcomes: the COMBINE study. Alcohol Clin Exp Res [Internet]. 2008 [acesso em 11 ago 2017]; 32(9). Disponível em: https://dx.doi.org/10.1111/j.15300277.2008.00743.x.

37. Weiss RD, Griffin ML, Potter JS, Dood DR, Dreifuss JA, Connery HS, et al. Who benefits from additional drug counseling among prescription opioid-dependent patients receiving buprenorphinenaloxone and standard medical management? Drug Alcohol Depend. [Internet]. 2014 [acesso em 17 out 2017]; (140). Disponível em: https://dx.doi.org/10.1016/j.drugalcdep.2014.04.005.

38. Burns L, Gisev N, Larney S, Dobbins T, Gibson A, Kimber J, et al. A longitudinal comparison of retention in buprenorphine and methadone treatment for opioid dependence in New South Wales, Australia. Addiction [Internet]. 2014 [acesso em 21 nov 2017]; 110(4). Disponível em: https://dx.doi. org/10.1111/add.12834.

39. Jaremko KM, Sterling RC, Van Bockstaele EJ. Psychological and physiological stress negatively impacts early engagement and retention of opioid-dependent individuals on methadone maintenance. J Subst Abuse Treat [Internet]. 2015 [acesso em: 19 nov 2017]; 48(1). Disponível em: https://dx.doi. 
40. Dunn K, DeFulio A, Everly JJ, Donlin WD, Aklin WM, Nuzzo PA, et al. Employment-based reinforcement of adherence to oral naltrexone in unemployed injection drug users: 12-month outcomes. Psychol Addict Behav [Internet]. 2015 [acesso em 03 out 2017]; 29(2). Disponível em: https://dx.doi. org/10.1037/adb0000010.

41. Campos RTO, Passos E, Palombini AL, Gonçalves LLM, Santos DVD, Melo SSJ, et al. Gestão autônoma da medicação. Guia de apoio a moderadores. 2014 [acesso em 03 nov 2017]. Disponível em: https://www.fcm.unicamp.br/fcm/sites/default/files/paganex/guia gam moderador - versao para download julho 2014.pdf.

42. Raffa JD, Grebely J, Tossonian H, Wong T, Viljoen M, Khara M, et al. The impact of ongoing illicit drug use on methadone adherence in illicit drug users receiving treatment for HIV in a directly observed therapy program. Drug Alcohol Depend. [Internet]. 2007 [acesso em 09 ago 2017]; 89(2-3). Disponível em https://doi.org/10.1016/j.drugalcdep.2007.02.007.

43. Parran TV, Adelman CA, Merkin B, Pagano ME, Defranco R, lonescu RA, et al. Long-term outcomes of office-based buprenorphine/naloxone maintenance therapy. Drug Alcohol Depend. [Internet]. 2010 [acesso em 18 set 2017]; 106(1). Disponível em: https://dx.doi.org/10.1016/j.drugalcolep.2009.07.013.

44. Neumann AM, Blondell RD, Azadfard M, Nathan G, Homish CG. Primary care patient characteristics associated with completion. Addict Behav [Internet]. 2013 [acesso em 10 out 2017]; 38(11). Disponível em: https://dx.doi.org/10.1016/j.addbeh.2013.07.007.

45. Shepherd A, Perella B, Hattingh HL. The impact of dispensing fees on compliance wih opioid substitution therapy: a mixed methods study. Subst abuse treat prev policy [Internet]. 2014 [acesso em 11 dez 2017]; 9(32). Disponível em: https://dx.doi.org/10.1186/1747-597X-9-32.

46. Corrêa Filho JM, Baltieri DA. Psychosocial and clinical predictors of retention in outpatient alcoholism treatment. Rev. Bras. Psquiatr. [Internet]. 2012 [acesso em 21 set 2017]; 34(4). Disponível em: https:// dx.doi.org/10.1016/j.rbp.2012.03.003.

47. Sohler NL, Li X, Kunins HV, Sacajiu G, Giovanniello A, Whitley S, et al. Home-versus office-based buprenorphine inductions for opioid-dependent patients. J subst abuse treat [Internet]. 2010 [acesso em 09 ago 2017]; 38(2). Disponível em: https://dx.doi.org/10.1016/j.jsat.2009.08.001.

48. Liu E, Liang $T$, Shen $L$, Zhong $H$, Wang B, Wu Z, et al. Correlates of methadone client retention: a prospective cohort study in Guizhou province, China. Int J Drug Policy [Internet]. 2009 [acesso em 18 ago 2017]; 20(4). Disponível em: https://dx.doi.org/10.1016/j.drugpo.2008.09.004.

49. Alford DP, LaBelle CT, Richardson JM, O'Connell JJ, Hohl CA, Cheng DM, et al. Treating homeless opioid dependent patients with buprenorphine in an office-based setting. J Gen Intern Med [Internet]. 2007 [acesso em 10 ago 2017]; 22(2). Disponível em: https://dx.doi.org/10.1007/s11606-006-0023-1.

50. Chen W, Hong Y, Zou X, McLaughlin MM, Xia Y, Ling L. Effectiveness of prize-based contingency management in a methadone maintenance program in China. Drug alcohol Depend. [Internet]. 2013 [acesso em 11 out 2017]; 133(1). Disponível em: https://dx.doi.org/10.1016/j.drugalcdep.2013.05.028.

51. Hartung D, Low A, Jindai K, Mansoor D, Judge M, Mendelson A, et al. Interventions to improve pharmacological adherence among adults with psychotic spectrum disorders end bipolar disorder: a systematic review. Psychosomatics. [Internet]. 2017 [acesso em 09 ago 2017]; 58(2). Disponível em: http:// dx.doi.org/10.1016/j.psym.2016.09.009.

52. American Psychiatric Association. DSM-5 Manual Diagnóstico e Estatístico de Transtornos Mentais. 5. ed. Porto Alegre: Artmed, 2014.

53. Sousa LPC, Vedana KGG, Miasso, Al. Adesão ao tratamento medicamentoso por pessoas com transtorno de ansiedade. Cogitare enferm. [Internet]. 2016 [acesso em 09 ago 2017]; 21(1). Disponível em: http://dx.doi.org/10.5380/ce.v21i1.43510. 
54. Almeida RA, Anjos UU, Vianna RPT, Pequeno GA. Perfil dos usuários de substâncias psicoativas de João Pessoa. Saúde debate [Internet]. 2014 [acesso em 09 ago 2017]; 38(102). Disponível em: http:dx.doi. org/10.5935/0103-1104.20140049.

55. Cruz VD, Oliveira MM, Pinho LB, Coimbra VCC, Kantorski LP, Oliveira JF. Condições sociodemográficas e padrões de consumo de crack entre mulheres. Texto contexto- enferm [Internet]. 2014 [acesso em 10 ago 2017]; 23(4). Disponível em: https://dx.doi.org/10.1590/010407072014000580013.

56. Pereira MV, Alencar JS, Souto RP, Pinto NB, Saraiva EMS. Grau de conhecimento dos pacientes sobre o tratamento: estratégia para uso racional de medicamentos. J Health NPEPS. [Internet]. 2016 [acesso em 10 ago 2017]; 1(1). Disponível em: https://periodicos.unemat.br/index.php/jhnpeps/article/view/1557.

57. Cortes LF, Terra MG, Pires FB, Heinrich J, Machado KL, Weiller, TH, et al. Atenção a usuários de álcool e outras drogas e os limites da composição de redes. Rev. Eletr. Enf. [Internet]. 2014 [acesso em 03 nov 2017]; 16(1). Disponível em: http://dx.doi.org/10.5216/ree.v16i1.20279.

58. Souza MSF, Kopittke L. Adesão ao tratamento com psicofármacos: fatores de proteção e motivos de não adesão ao tratamento farmacológico. Rev. APS. [Internet]. 2016 [acesso em 3 nov 2017]; 19(3). Disponível em: https://aps.ufjf.emnuvens.com.br/aps/article/view/2370/1012.

Recebido: 15/04/2018

Finalizado: 16/01/2019

Autor Correspondente:

Mariluci Alves Maftum

Universidade Federal do Paraná

R. Lothario Meisser, 632, Bloco Didático II - 80210-170 - Curitiba, PR, Brasil

E-mail: maftum@ufpr.br

Contribuição dos autores:

Contribuições substanciais para a concepção ou desenho do estudo; ou a aquisição, análise ou interpretação de dados do estudo - FCC

Elaboração e revisão crítica do conteúdo intelectual do estudo - ACZF, CBA, GJM

Aprovação da versão final do estudo a ser publicado - MAM

Responsável por todos os aspectos do estudo, assegurando as questões de precisão ou integridade de qualquer parte do estudo - FCC, MAM 